\title{
Análisis in silico del gen subolesina como posible vacuna contra garrapatas Rhipicephalus microplus
}

\section{In silico analysis of the subolesin gene as a possible vaccine against $R$ hipicephalus microplus ticks}

\author{
José Octavio Merino-Charrez ${ }^{1}\left[\right.$, Ninnet Gómez-Romero²], Ivette Barrera-Molina ${ }^{3}$, Rodolfo \\ Lagunes-Quintanilla* ${ }^{4 *}$ \\ ${ }^{1}$ Facultad de Medicina Veterinaria y Zootecnia, Universidad Autónoma de Tamaulipas, Carretera Victoria-Mante, Km. 5, CP. 87000. \\ Ciudad Victoria, Tamaulipas, México. \\ ${ }^{2}$ Facultad de Medicina Veterinaria y Zootecnia, Universidad Nacional Autónoma de México. Avenida Universidad 3000, CP. 04510. \\ Ciudad de México, México. \\ ${ }^{3}$ Facultad de Nutrición, Universidad Nacional Autónoma del Estado de Morelos. Calle Iztaccíhuatl 100, Col. Los Volcanes, CP. 62350. \\ Cuernavaca, Morelos, México. \\ ${ }^{4}$ Centro Nacional de Investigación Disciplinaria en Parasitología Veterinaria, Instituto Nacional de Investigaciones Forestales, Agrícolas y \\ Pecuarias. Carretera Federal Cuernavaca-Cuautla 8534, Col. Progreso, CP. 62550. Jiutepec, Morelos, México. \\ *Autor de correspondencia: rodolfo.lagunes@gmail.com
}

Nota científica recibida: 10 de mayo de 2018 aceptada: 02 de octubre de 2018

RESUMEN. El objetivo del estudio fue utilizar estrategias bioinformáticas para predecir propiedades antigénicas de interés del gen subolesina, así como realizar construcciones filogenéticas para identificar la relación que existe entre los aislados geográficos de garrapatas reportados en el mundo. El gen subolesina presenta $98 \%$ de identidad entre los diferentes aislados de garrapatas Rhipicephalus microplus, mientras que, entre diferentes géneros (Ixodes spp., Amblyomma spp., Hyalomma spp.) presenta un menor grado de identidad (77\%). Se detectaron cuatro epítopos lineales de células B conservados en diferentes especies de garrapatas. El análisis de la estructura secundaria de la proteína Subolesina mostró dos $\alpha$-hélices, tres regiones inespecíficas (coil) y ninguna $\beta$ plegada. De los epítopos predichos, se debe demostrar su capacidad inmunoprotectora para el desarrollo de un inmunógeno efectivo contra poblaciones de garrapatas $R$. microplus.

Palabras clave: Bioinformática, ectoparásitos, filogenia, inmunógeno, Rhipicephalus microplus.

ABSTRACT. The objective was to use bioinformatic strategies to predict antigenic properties of interest of the subolesin gene, as well as to carry out phylogenetic constructions to identify the relationship that exists between the geographic isolates of ticks reported in the world. The subolesin gene has $98 \%$ identity among the different Rhipicephalus microplus tick isolates, while among different genera (Ixodes spp., Amblyomma spp., Hyalomma spp.) it has a lower degree of identity (77\%). Four linear epitopes of B cells conserved in different tick species were detected. Analysis of the secondary structure of the Subolesin protein showed two $\alpha$-helixes, three coil regions and no folded $\beta$. Of the predicted epitopes, their immunoprotective ability must be demonstrated for the development of an effective immunogen against $R$. microplus tick populations.

Key words: Bioinformatics, ectoparasites, phylogeny, immunogen, Rhipicephalus microplus.

\section{INTRODUCCIÓN}

La garrapata Rhipicephalus microplus es el principal ectoparásito que afecta a la ganadería bovina en regiones tropicales y subtropicales del mundo. Los efectos causados por las infestaciones representan uno de los principales problemas que repercuten en la productividad de los bovinos, sin olvidar el papel que juegan en la transmisión de enfermedades como babesiosis (Babesia bovis, $B$. bigemina) y anaplasmosis (Anaplasma marginale) (Almazán et al. 2018). Se estima que el costo global ocasionado por las garrapatas y las enfermedades que transmiten oscila entre los 2.5 billones de dólares 
anuales (Lew-Tabor et al. 2014). El método de control más utilizado para contrarrestar los efectos causados por estos ectoparásitos ha sido por mucho tiempo el control químico, pero el uso constante de los ixodicidas ha favorecido el desarrollo de garrapatas resistentes (Rodríguez-Vivas et al. 2014). Desafortunadamente, en la actualidad se han detectado cepas de garrapatas que presentan resistencia a diversos ixodicidas, lo que dificulta su control (Robbertse et al. 2016). Por lo cual, el control inmunológico representa una alternativa promisoria, debido a que ha logrado disminuir la infestación y bloquear la transmisión de patógenos a sus hospedadores (Almazán et al. 2018). El antígeno Bm86, es el componente de las vacunas comerciales contra garrapatas que existen; estas vacunas disminuyen los parámetros reproductivos de las garrapatas, afectando con ello la progenie. Sin embargo, su eficacia es variable dependiendo de las cepas o aislados geográficos (GarcíaGarcía et al. 1999, Popara et al. 2013).

La proteína Subolesina, descubierta en la garrapata Ixodes scapularis (Almazán et al. 2010), se encuentra presente en los diferentes estadios de la garrapata y en varios órganos como intestino, glándulas salivales y ovarios (Merino et al. 2013). Actualmente, se sabe que es eficaz como antígeno protector, y es estructural y funcionalmente ortólogo de las akirinas en insectos y vertebrados (ManzanoRoman et al. 2012, Sultana et al. 2015). Por ello, se han desarrollado inmunógenos que contienen el antígeno subolesina que induce protección inmunológica en contra de las infestaciones de garrapatas en hospedadores vertebrados. A la fecha, subolesina es considerado como un candidato vacunal de importancia (Merino et al. 2013, Shakya et al. 2014, Sultana et al. 2015), ya que la inmunización de los animales, genera protección cruzada frente a diversas especies de vectores hematófagos, sugiriendo la posibilidad de usar subolesina como antígeno para desarrollar una vacuna universal para diversos organismos. Por lo anterior, el objetivo fue utilizar herramientas basadas en la genómica, proteómica y bioinformática para analizar el gen subolesina de diferentes aislados geográficos, con la finalidad de diseñar un antígeno vacunal con regiones conser- vadas e inmunogénicas que confieran protección eficiente contra distintas cepas de garrapatas (R. microplus).

\section{MATERIALES Y MÉTODOS}

Se utilizó la base de datos del GenBank para obtener las secuencias del gen subolesina de $R$. microplus, procedente de diferentes aislados geográficos, así como de distintos géneros de garrapatas. Las secuencias obtenidas se compararon realizando un alineamiento múltiple con el programa CLUSTAL W (Thompson et al. 1994), para determinar su identidad genética. Se realizó una matriz de sustitución para obtener el grado de identidad y similitud entre aislados geográficos de $R$. microplus, con el servidor SIAS diseñado por la Universidad Complutense de Madrid. El análisis filogenético se realizó con el software MEGA 6 (Tamura et al. 2013), para el cual se consideraron los siguientes parámetros: como modelo de sustitución nucleotídica, se utilizó el método Tamura de 3 parámetros (Tamura 1992), elegido con base en el valor del criterio de información Bayesiana. Como método de reconstrucción filogenética se utilizó el método de máxima verosimilitud (Guindon y Gascuel 2003) y para soporte estadístico se utilizaron 1000 réplicas de bootstrap (Felsenstein 1985). Se consideró la secuencia del gen subolesina de la garrapata Ixodes scapularis como grupo externo.

La secuencia que codifica para la proteína Subolesina de R. microplus cepa "Tamaulipas", México (Número de acceso AGI44615.1) se analizó con diferentes programas bioinformáticos para predecir propiedades de interés. Se utilizó el programa Protscale (Gasteiger et al. 2005), para predecir regiones hidrofóbicas e hidrofílicas. Para los análisis de las estructuras secundarias ( $\alpha$-helices y $\beta$ plegadas) se utilizó el programa PSIPRED (Buchan et al. 2013) y predicción del potencial antigénico se realizó con el método de Kolaskar y Tongaonkar (1990) con el programa Antigenic. La predicción de epítopos B se realizó con los servidores BcePred y ABCpred (Saha y Raghava 2004, 2006). Para el servidor BcePred se determinó un índice de $2.38 \%$, al considerar que este 
algoritmo posee diferentes valores de corte para cada una de las propiedades fisicoquímicas seleccionadas a partir de las cuales los péptidos predichos son considerados como epítopos. Para el servidor de ABCpred se seleccionaron epítopos de células $B$ de 12 aminoácidos de longitud con un índice de inmunidad de $0.5 \%$.

\section{RESULTADOS Y DISCUSIÓN}

Se obtuvieron del GenBank 66 secuencias que codifican para la proteína Subolesina, reportadas en Brasil, China, India, Laos, México, Mozambique y Estados Unidos (Figura 1). La reconstrucción del árbol filogenético muestra cuatro clados definidos, que corresponden a los géneros: Rhipicephalus spp., Hyalomma spp., Amblyomma spp., Ixodes spp. El $73 \%$ de las secuencias analizadas se agrupan dentro del clado de $R$. microplus mostrando una identidad del $98 \%$ entre los aislados geográficos. Los resultados sugieren que la longitud de la proteína Subolesina presenta diferentes procesos evolutivos que se reflejan en aspectos morfológicos, fisiológicos y genéticos; dando como resultado variabilidad en la longitud de las secuencias aminoacídicas, siendo en Ixodes spp. más largas que en Amblyomma spp., Hyalomma spp. y Rhipicephalus spp.

Para generar la matriz de identidad/similitud (Tabla 1), se seleccionaron 23 secuencias del gen subolesina de distintos aislados geográficos de garrapatas. El análisis mostró valores del 98 al 100\% para la especie $R$. microplus. Los resultados revelan alto grado de conservación y por ende una estructura similar entre los diferentes aislados; además, los valores de identidad/similitud al compararse con el grupo ortólogo, mostraron una identidad de $77 \%$. Con el propósito de predecir regiones antigénicas y epítopos lineales de células $B$, en la secuencia de la proteína Subolesina; se realizó un análisis con las herramientas bioinformáticas BcePred y ABCpred. La predicción sugiere que existen cuatro epítopos $\mathrm{B}$ a lo largo de la secuencia de aminoácidos con un índice de inmunidad superior a $0.8 \%$, lo que es indicativo de alta inmunogenicidad. Con respecto a la topología, posición y localización de los péptidos predichos, se presume que se encuentran dentro de estructuras $\alpha$ hélices o muy cercanos a ellas (Figura 2). Los resultados indican que la proteína Subolesina podría ser un candidato vacunal para el control de garrapatas en distintas regiones del mundo.

La literatura indica que Subolesina es una proteína de interés, que protege contra diferentes especies de artrópodos (Almazán et al. 2018). En ensayos de inmunización de bovinos contra garrapatas se ha observado una eficacia del 40 al 60\% (Almazán et al. 2010, Merino et al. 2013, Shakya et al. 2014), por lo que es un antígeno atractivo para el control de garrapatas. Actualmente se utilizan diferentes ramas de la bioinformática, como la inmunoinformática, que se basa en la aplicación de herramientas computacionales al estudio de las moléculas del sistema inmune, capaz de guiar el diseño de experimentos para responder a importantes interrogantes en la inmunobiología y la vacunología (Tomar y De 2010). La predicción de epítopos lineales de células $\mathrm{B}$ podría disminuir en gran medida el trabajo experimental en la búsqueda de candidatos vacunales, como se ha hecho en otros modelos de investigación (Vaughan et al. 2010, Mahajan et al. 2010). Se detectaron cuatro epítopos lineales de células $B$ tomando en cuenta únicamente la región extracelular de la proteína, por lo que sería útil demostrar la capacidad inmunoprotectora de dichos epítopos en ensayos de inmunización de bovinos contra garrapatas $R$. microplus. Si la premisa de que son inmunoprotectores se cumple, sería una ventaja utilizarlos para el desarrollo de una vacuna contra garrapatas, considerando que no existe variabilidad en la proteína. 
Merino-Charrez et al.

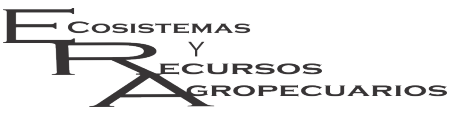

Análisis bioinformático del gen subolesina

Ecosist. Recur. Agropec.

6(16):129-136,2019

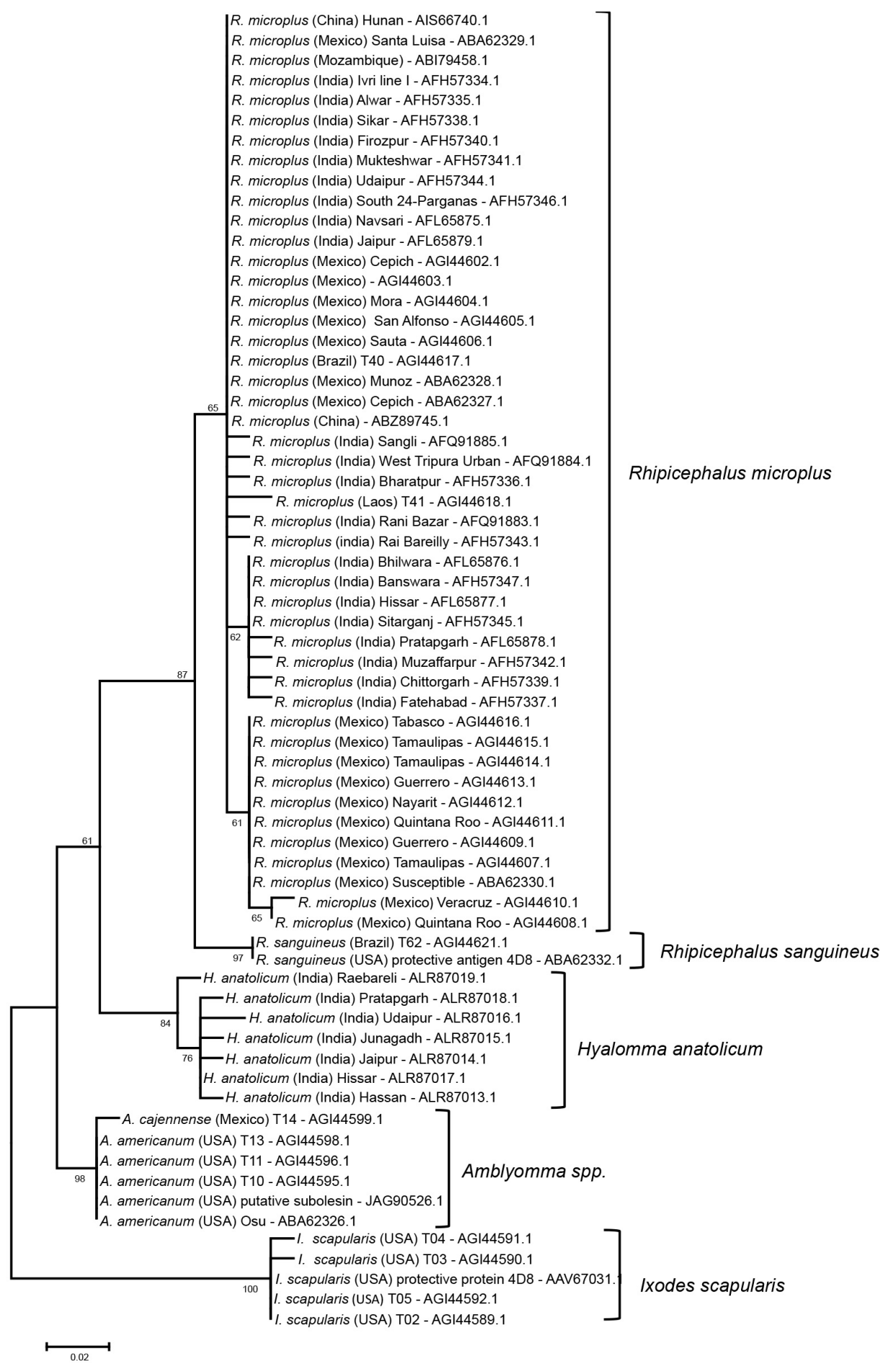

Figura 1. Análisis filogenético de la proteína Subolesina. El árbol fue construido por el método de máxima verosimilitud con secuencias de referencia de distintas especies de garrapatas obtenidas del GenBank. Los números en los nodos indican valores de bootstrap y probabilidad posterior del análisis Bayesiano, cada secuencia se identifica con su origen y número de acceso. 


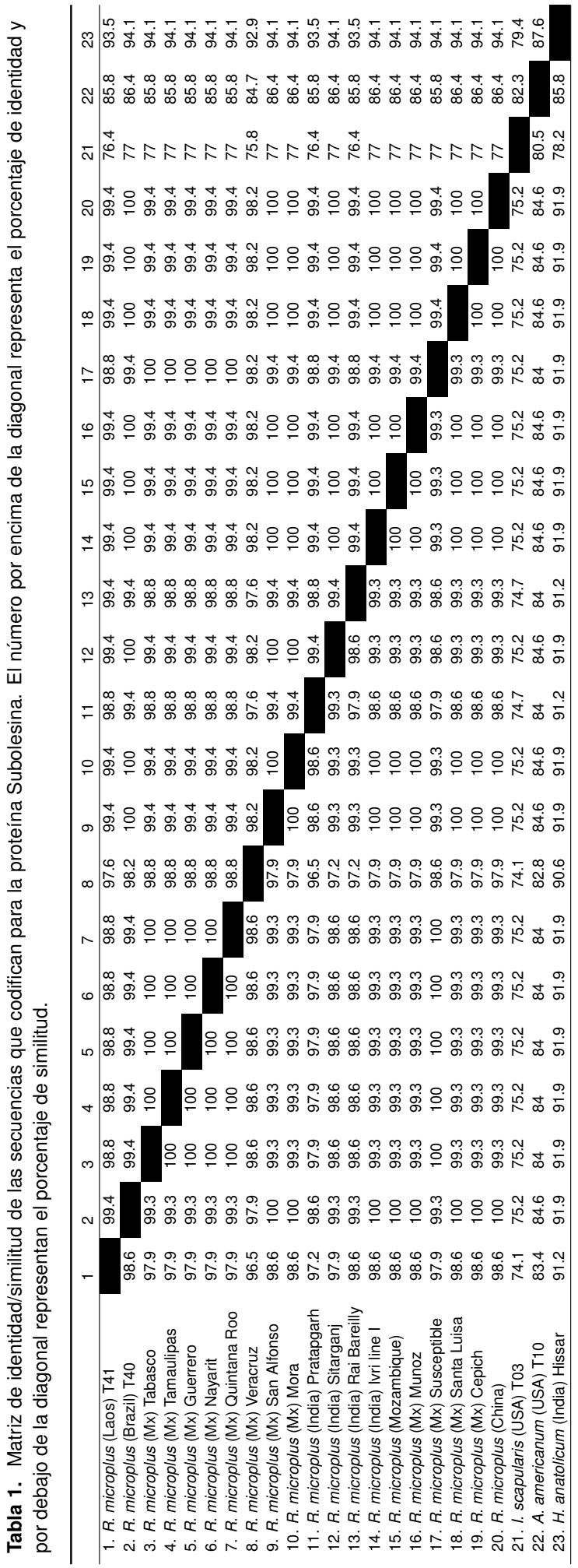




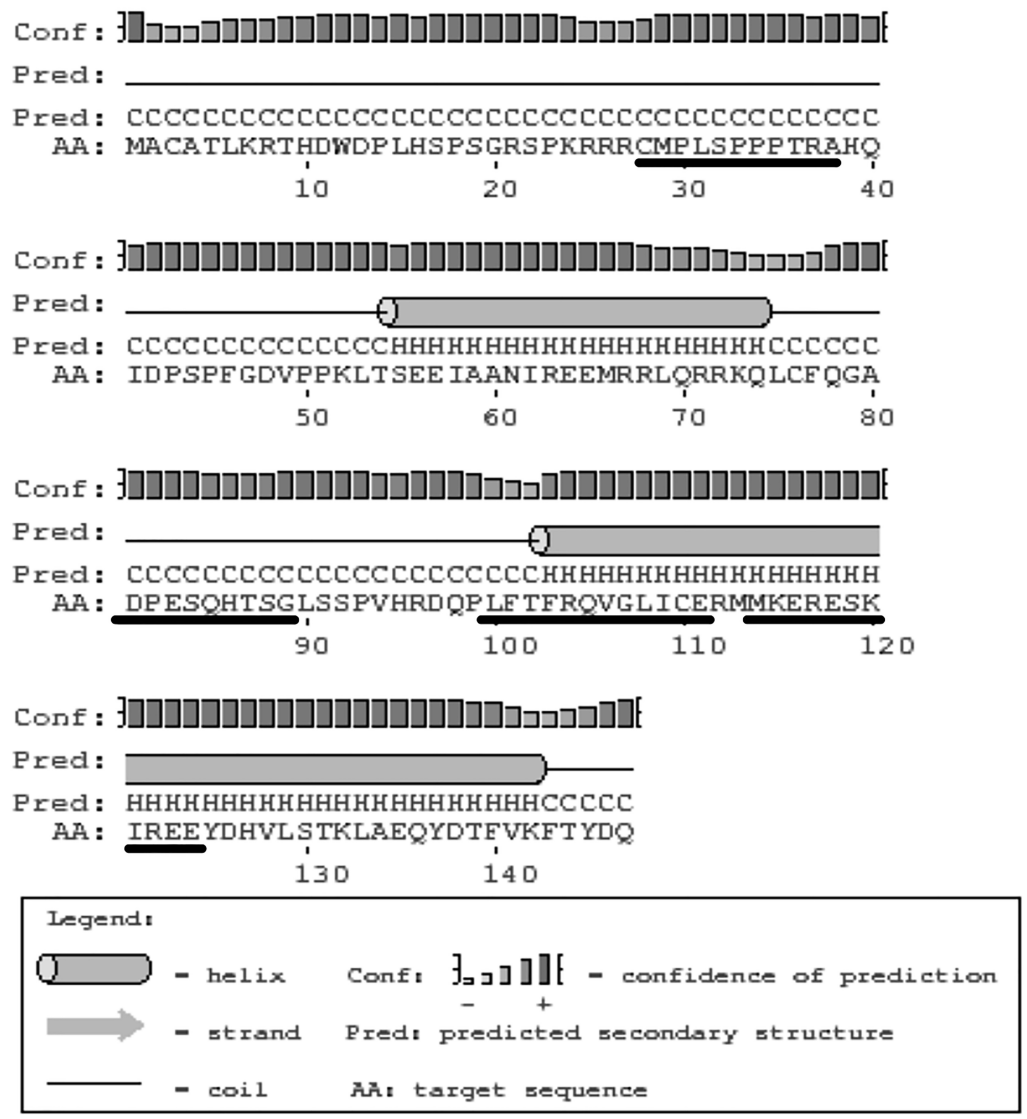

Figura 2. Análisis de predicción de la estructura secundaria de Subolesina. Los cilindros representan estructuras $\alpha$-hélices (helix), las flechas indican estructuras lámina beta (strand) y las líneas en negro son los residuos que no tienen una estructura secundaria definida (coil). En color negro subrayado se muestran los epítopos lineales de células B.

\section{LITERATURA CITADA}

Almazán C, Lagunes R, Villar M, Canales M, Rosario-Cruz R, Jongejan F, et al. (2010) Identification and characterization of Rhipicephalus (Boophilus) microplus candidate protective antigens for the control of cattle tick infestations. Parasitology Research 106: 471-479.

Almazán C, Aguilar-Tipacamu G, Rodríguez S, Mosqueda J, Pérez de León AA (2018) Immunological control of ticks and tick-borne diseases that impact cattle health and production. Frontiers in Bioscience 23: 15351551.

Buchan DWA, Minneci F, Nugent TCO, Bryson K, Jones DT (2013) Scalable web services for the PSIPRED Protein Analysis Workbench. Nucleic Acids Research 41: W340-W348.

Felsenstein J (1985) Phylogenies and the Comparative Method. The American Naturalist 125: 1-15.

García-García JEC, González IL, González DM, Valdés M, Méndez L, Lamberti J, et al. (1999) Sequence variations in the Boophilus microplus Bm86 locus and implications for immunoprotection in cattle vaccinated with this antigen. Experimental Applied Acarology 23: 883-895. 
Gasteiger E, Hoogland C, Gattiker A, Duvaud S, Wilkins MR, Appel RD, et al. (2005) Protein identification and analysis tools on the ExPASy server. In: Walker JM (ed): The Proteomics protocols handbook. Humana Press 571-607.

Guindon S, Gascuel O (2003) A simple, fast, and accurate algorithm to estimate large phylogenies by Maximum Likelihood. Systematic Biology 52: 696-704.

Kolaskar AS, Tongaonkar PC (1990) A semi-empirical method for prediction of antigenic determinants on protein antigens. FEBS Letters 276: 172-174.

Lew-Tabor AE, Bruyeres AG, Zhang B, Valle MR (2014) Rhipicephalus (Boophilus) microplus tick in vitro feeding methods for functional (dsRNA) and vaccine candidate (antibody) screening. Ticks Tick Borne Diseases 5: 500-510.

Mahajan B, Berzofsky JA, Boykins RA, Majam V, Zheng H, Chattopadhyay R, et al. (2010) Multiple antigen peptide vaccines against Plasmodium falciparum malaria. Infection and Immunity 78: 4613-4624.

Manzano-Roman R, Diaz-Martin V, Oleaga A, Siles-Lucas M, Perez-Sanchez R (2012) Subolesin/akirin orthologs from Ornithodoros spp. soft ticks: cloning, RNAi gene silencing and protective effect of the recombinant proteins. Veterinary Parsitology 185: 248-59.

Merino O, Antunes S, Mosqueda J, Moreno-Cid JA, Pérez de la Lastra JM, Rosario-Cruz R, et al. (2013) Vaccination with proteins involved in tick-pathogen interactions reduces vector infestations and pathogen infection. Vaccine 31: 5889-5896.

Popara M, Villar M, Mateos-Hernández L, Fernández de Mera IG, Marina A, del Valle M, et al. (2013) Lesser protein degradation machinery correlates with higher BM86 tick vaccine efficacy in Rhipicephalus annulatus when compared to Rhipicephalus microplus. Vaccine 31: 4728-4735.

Robbertse L, Baron S, Van Der Merwe NA, Madder M, Stoltsz WH, Maritz-Olivier C (2016) Genetic diversity, acaricide resistance status and evolutionary potential of a Rhipicephalus microplus population from a diseasecontrolled cattle farming area in South Africa. Ticks and Tick Borne Diseases 7: 595-603.

Rodríguez-Vivas R, Pérez-Cogollo L, Rosado-Aguilar J, Ojeda-Chi M, Trinidad-Martínez I, Miller R, et al. (2014) Rhipicephalus (Boophilus) microplus resistant to acaricides and ivermectin in cattle farms of Mexico. Brazilian Journal of Veterinary Parasitology 2961: 113-122.

Saha S, Raghava GPS (2004) BcePred: Prediction of Continuous B-Cell Epitopes in Antigenic Sequences Using Physico-chemical Properties. In: Nicosia G, Cutello V, Bentley PJ, Timis J (eds.) ICARIS. LNCS 3239: 197-204.

Saha S, Raghava GPS (2006) Prediction of continuous B-cell epitopes in an antigen using recurrent neural network. Proteins 65: 40-48.

Shakya M, Kumar B, Nagar G, de la Fuente J, Ghosh S (2014) Subolesin: a candidate vaccine antigen for the control of cattle tick infestations in Indian situation. Vaccine 32: 3488-3494.

Sultana H, Patel U, Sonenshine DE, Neelakanta G (2015) Identification and comparative analysis of subolesin/akirin ortholog from Ornithodoros turicata ticks. Parasites \& Vectors 8: 132.

Tamura K (1992) Estimation of the number of nucleotide substitutions when there are strong transition-transversion and $\mathrm{G}+\mathrm{C}$ content biases. Molecular Biology and Evolution 9: 678-687.

Tamura K, Stecher G, Peterson D, Filpiski A, Kumar S (2013) MEGA6: Molecular Evolutionary Genetics Analysis versión 6.0. Molecular Biology and Evolution 30: 2725-2729. 


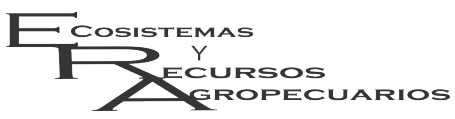

Thompson JD, Higgins DG, Gibson TJ (1994) Clustal W; improving the sensitivity of progressive multiple sequence aligment trough sequence weighting, position-specific gap penalties and weight matriz choice. Nucleic Acids Research 22: 4673-4680.

Tomar N, De RK (2010) Immunoinformatics: an integrated scenario. Immunology 131: 153-168.

Vaughan K, Greenbaurn J, Blythe M, Peters B, Sette A (2010) Metaanalysis of all immune epitope data in the Flavivirus genus: inventory of current immune epitope data status in the context of virus immunity and immunopathology. Viral Immunology 23: 259-284. 